\title{
PROSPECTIVE OBSERVATIONAL STUDY ON ANTIBIOTIC-PRESCRIBING PATTERN AND MEDICATION ERRORS IN SURGICAL PROPHYLAXIS IN A SPECIALTY HOSPITAL
}

\author{
SENTHIL KUMAR N, SIVASAKTHI K*, JISA ELIZABETH JOSEPH, VISHNUKUMAR VA, GEOLIN RP
}

Jkkmmrf College Of Pharmacy.India. Email: sakthi.siva1292@gmail.com

Received: 30 October 2019, Revised and Accepted: 05 December 2019

ABSTRACT

Objective: The objective was to study the antibiotic-prescribing patterns, identify the medication errors and impact of surgical antimicrobial prophylaxis (SAP) in preventing surgical site infection (SSI), and to understand the prescribers' adherence to surgical prophylaxis guidelines.

Methods: The study was conducted for a period of 6 months in all surgical departments of a specialty hospital. Data were collected from inpatients records. Australian guideline for SAP was used to assess the appropriateness in prescribing pattern. The sample size was calculated using Raosoft sample size calculator.

Results: A prospective observational study was carried out among 178 patients. Of which, 100 were male and 78 were female. Four hundred and thirtythree antimicrobials were prescribed as pre- and post-operative surgical prophylaxis, among that $87 \%$ prescribed by brand name and $13 \%$ by generic. Seventy-one percent received single antimicrobial agent preoperatively, of which $99.5 \%$ prescribed as parenteral and $0.5 \%$ as oral formulation. Most often prescribed antibiotic was cefoperazone (28\%) of cephalosporin group. Only 5.6\% of cases had compliance with SAP guidelines. In this study, 11 patients affected with SSI due to inappropriate antibiotic selection and non-adherence to prophylactic antibiotic guidelines.

Conclusion: The present study revealed that there is a poor compliance to SAP guidelines in terms of inappropriateness in antibiotic drug selection, dose, duration, and omission of drugs. Inappropriateness and non-compliance are mainly due to unavailability of clinical pharmacist to assist the physicians in the selection and administration of correct choice of prophylactic drug and unavailability of proper national or local guidelines. Hence, there is dire need to make local SAP guidelines to improve SAP-prescribing pattern.

Keywords: Surgical antibiotic prophylaxis, Surgical site infection, Surgical antimicrobial prophylaxis guidelines surgical wounds.

(c) 2020 The Authors. Published by Innovare Academic Sciences Pvt Ltd. This is an open access article under the CC BY license (http://creativecommons. org/licenses/by/4. 0/) DOI: http://dx.doi.org/10.22159/ajpcr.2020.v13i2.36212

\section{INTRODUCTION}

Surgical site infection (SSI) is one of the most often post-operative complications and represents a notable burden in terms of patient morbidity and mortality [1]. SSIs usually occur at or near the incision area within 30 or 90 days, depending upon the procedure performed. SSIs are often localized to the incision site but can also extend into deeper adjacent structures [2].

Surgical antibiotic prophylaxis plays a major role in preventing occurrence of SSI after procedure. Appropriate antimicrobial agent (AMA) selection mainly depends on the pathogens most likely to cause an infection. Narrow-spectrum antibiotics are often used as pre-operative prophylactic agent because in which most organisms responsible for hospital-acquired infections are covered. The selection of antibiotic with the narrowest antibacterial spectrum is required to reduce the occurrence of multiresistant pathogens and also because broad-spectrum antibiotics may be required later if the patients develop series sepsis. Therefore the use of 'third generation' cephalosporins such as ceftriaxone and cefotaxime should be avoided as surgical prophylaxis due to its lower efficacy in preventing SSI [3]. Cefazolin is considered as a primary choice of antibiotic for surgical prophylaxis due to its greater effectiveness toward methicillin-resistant Staphylococcus aureus and methicillinsusceptible $S$. aureus infection. In some cases, especially for surgery $>4 \mathrm{~h}$, redosing after $4 \mathrm{~h}$ is necessary to maintain the plasma drug concentration. In appendectomy/colorectal surgery, cefazolin+metronidazole-like drugs are needed for its better effectiveness because they are more prone to anaerobic infection at surgical site. Parenteral secondgeneration cephalosporins such as cefotetan are sometimes used as a more convenient antibiotic compared to first-generation cephalosporins because of its improved anaerobic and aerobic Gram-negative coverage.
These are also used as an alternative to the combination of metronidazole plus either first-generation cephalosporins or gentamicin for abdominal surgical prophylaxis, but they are more expensive. The bacterial flora in some hospitalized patients may include multiresistant bacteria such as methicillin-resistant staphylococci. In such cases, vancomycin is added with cephalosporins to prevent the post-operative SSI. Apart from drug selection, timing also has importance. Surgical antimicrobial prophylaxis (SAP) must be started within $1 \mathrm{~h}$ before incision. In case of patients receiving vancomycin, it must be administered within $2 \mathrm{~h}$ before surgery. Prophylactic antibiotic should be discontinued within $24 \mathrm{~h}$ of surgery completion. However, in case of cardiothoracic surgery, it must be continued for $48 \mathrm{~h}$ [4].

\section{METHODS}

A prospective observational study was conducted in a surgical ward in all departments of a specialty hospital in Salem, Tamil Nadu, for a period of 6 months from February 2019 to July 2019. During the study period, patients who underwent surgery and met our inclusion criteria were conveniently selected, and the study population (178 patients) was calculated using Raosoft sample size calculator.

The baseline data collection was done by case sheet analysis, and further information was collected from either patient or their caretakers and recorded on data collection form which was previously prepared. The patient admitted for $<3$ days and those not willing to provide signed consent and patients who died or referred to higher centers were excluded from our study.

The data were collected in three parts. The first part includes patient demographic data (age, gender, and date of admission and discharge), 
Table 1: Classification of antibiotics administered before surgery

\begin{tabular}{|c|c|c|c|c|c|c|}
\hline \multirow[t]{2}{*}{ S. No. } & \multirow[t]{2}{*}{ Prophylactic antibiotic group } & \multirow[t]{2}{*}{ Prophylactic antibiotic } & \multicolumn{2}{|l|}{ Administer as alone } & \multicolumn{2}{|c|}{ Administer as combination } \\
\hline & & & Frequency $(n=204)$ & Percentage & Frequency $(n=204)$ & Percentage \\
\hline \multirow[t]{3}{*}{1.} & Cephalosporins & Cefoperazone & 47 & 23 & 10 & 5 \\
\hline & & Ceftriaxone & 25 & 12.2 & 9 & 4.4 \\
\hline & & Cefuroxime & 9 & 4.4 & 4 & 2 \\
\hline \multirow[t]{2}{*}{2.} & Penicillins & Amoxicillin & 6 & 2.9 & 1 & 0.5 \\
\hline & & Piperacillin & 11 & 5.4 & 3 & 1.5 \\
\hline \multirow[t]{2}{*}{3.} & Aminoglycosides & Amikacin & 6 & 2.9 & 1 & 0.5 \\
\hline & & Gentamycin & - & - & 1 & 0.5 \\
\hline 4. & Nitroimidazoles & Metronidazole & 1 & 0.5 & 22 & 10.8 \\
\hline 5. & Fluoroquinolone & Ofloxacin & - & - & 5 & 2.4 \\
\hline \multirow[t]{2}{*}{6.} & Carbapenems & Meropenem & 2 & 1 & 2 & 1 \\
\hline & & Imipenem & - & - & 1 & 0.5 \\
\hline 7. & Polypeptides & Colistin & - & - & 2 & 1 \\
\hline 8. & Oxazolidinones & Linezolid & 1 & 0.5 & - & - \\
\hline 9. & Glycylcyclines & Tigecycline & - & - & 1 & 0.5 \\
\hline \multicolumn{3}{|c|}{ Grand total (frequency) } & 204 & & & \\
\hline
\end{tabular}

comorbidity, allergic status, and vital signs. The second part includes information regarding surgical procedures, type of wound, antibiotics prescribed, and details regarding culture sensitivity test. In the third part, details regarding medication errors in antibiotic-prescribing pattern were included. Inappropriateness in prescription was analyzed using Australian guidelines for surgical antibiotic prophylaxis.

\section{Ethical considerations}

The prescription analysis was done in compliance with Australian guidelines after obtaining approval from the institutional ethical committee and the hospital management.

\section{RESULTS AND DISCUSSION}

A prospective observational study was carried out among 178 patients from the time of admission till their first review. A total of 178 patients were recruited, of which 100 patients were male and rests (78) were female. Most frequent cases fell under the age category between 51 and 65 years $(27.5 \%)$, followed by $19-35(26.4 \%), 36-50(22.5 \%)$, and others with a mean duration of hospital stay of $6.03 \pm 3.05$. Most often, patients $(63.5 \%)$ undergone surgery without any comorbid conditions and $13.5 \%$ had diabetes mellitus with hypertension (HTN). Majority cases admitted in general department (28.7\%), followed by gynecology/obstetrics (23\%), orthopedic (21.9\%), and least frequent in neurology. Majority of cases reviewed were targeted $(73.6 \%)$.

Only for nine cases, antibiotics were prescribed after doing antibiotic sensitivity and resistance test. One of the errors noted in this study was the administration of resistant/allergic drug to around ten patients. Most of the surgical wound were clean contaminated (44.9\%), followed by clean (36.5\%), dirty (13.5\%), and contaminated (5.1\%). Povidoneiodine was most often used for skin preparation before incision.

Among the total population, 162 received pre-operative antibiotics. In which, $71 \%$ received single-drug therapy, followed by double therapy (18.5\%). Post-operative administration switched from 162 to 168 patients, of which single therapy (68\%) constitutes the major portion. Ten cases undergone surgery without any prophylactic drugs.

A total of 433 antimicrobials administered as pre- and post-operative drug. About $86.6 \%$ of drugs prescribed by brand name and $13.4 \%$ by generic name.

Cefoperazone of cephalosporin group is prescribed to $28 \%$ of cases. The second most class of drug prescribed was nitroimidazoles (11.3\%). According to standard guideline, cefazolin is considered as the primary
Table 2: Evaluation of prophylactic antibiotic administration

\begin{tabular}{llll}
\hline S. No. & Prophylactic antibiotic & Frequency $(\mathbf{n = 1 7 8})$ & Percentage \\
\hline 1. & $\begin{array}{l}\text { Required and } \\
\text { administered }\end{array}$ & 88 & 49.4 \\
2. & $\begin{array}{l}\text { Required but not } \\
\text { administered }\end{array}$ & 80 & 44.9 \\
$3 . \quad \begin{array}{l}\text { Not required but } \\
\text { administered }\end{array}$ & 9 & 5.1 \\
$4 . \quad \begin{array}{l}\text { Not required and not } \\
\text { administered }\end{array}$ & 1 & 0.6 \\
Total & 178 & 100 \\
\hline
\end{tabular}

Table 3: Antibiotic administration pattern

\begin{tabular}{|c|c|c|c|}
\hline S. No. & Antibiotic administration & $\begin{array}{l}\text { Frequency } \\
(n=435)\end{array}$ & Percentage \\
\hline 1. & $\begin{array}{l}\text { Correct choice+correct } \\
\text { dose+correct time+correct } \\
\text { continuation }\end{array}$ & 10 & 2.3 \\
\hline 2. & $\begin{array}{l}\text { Correct choice+correct } \\
\text { dose+correct duration }\end{array}$ & 12 & 2.8 \\
\hline 3. & Correct choice+correct dose & 20 & 4.6 \\
\hline 4. & Correct choice of antibiotic & 21 & 4.8 \\
\hline
\end{tabular}

choice of antibiotic for surgical prophylaxis, but no one is treated with respective drug in this study.

A study conducted by Sharma and Goel [6] in 2018 reported that the most common group was the third generation cephalosporins (61\%), whereas individually, amikacin (58.5\%) was the most commonly prescribed individual AMA, followed by metronidazole $(55 \%)$ and ceftriaxone (44.5\%).

The timing of antibiotic administration also plays a major role in preventing SSI related to surgery. In this study, the antibiotic administration was proper in around $64.2 \%$.

Around $44.9 \%$ of cases require antibiotics before surgery, but not administered and nine cases not required antibiotics, but it was administered. This indicates the inappropriateness in the antibioticprescribing pattern and suggests that surgeons in our country are not aware about the importance of prophylaxis administration. About 49.4\% show appropriateness in SAP administration. 
Vessal et al. [7], 2019, reported that prophylactic antibiotics were administered in $98 \%$ of the procedures, whereas only $68 \%$ of the surgeries required them according to guidelines. Vessel suggests that the surgeons in Iran are aware of the value of antibiotics in preventing SSI.

Another error noted was the omission of drugs in both pre- and postoperatively. About $30.3 \%$ of prescriptions omit both pre- and post-operative drug. Antibiotic administration pattern in surgeries required prophylaxis which demonstrates the prescribing pattern and prescribers' knowledge about SAP prophylaxis.

Apart from 433 antibiotics, two more antibiotics administered to patients as intraoperative dose in a prolonged surgery of around $9 \mathrm{~h}$. Only $2.3 \%$ of cases satisfy all the recommendations noted in guidelines, and rest shows abnormalities in either antibiotic selection, timing or dose of administration.

Vessal et al. [7] reported that only 7.5\% were received correct drug with correct dose. Only $0.9 \%$ was prescribed according to guideline.

Table 4: Surgical prophylaxis compliance

\begin{tabular}{llll}
\hline S. No. & SAP compliance & Frequency $(\mathbf{n = 1 7 8})$ & Percentage \\
\hline 1. & According to guideline & 10 & 5.6 \\
2. & $\begin{array}{l}\text { Not according to } \\
\text { guideline }\end{array}$ & 168 & 94.3 \\
Total & & 178 & 100 \\
\hline
\end{tabular}

SAP: Surgical antimicrobial prophylaxis
The intervention of this study is that majority of cases were noncompliance with guidelines in terms of drug selection, dose, and timing. The details regarding prescriber compliance to SAP guideline are shown in Table 4.

In this study, 94.3\% were non-compliance with guideline due to lack of knowledge about prophylactic guidelines. Only 5.6\% were prescribed according to guideline and satisfy all criteria mentioned in guidelines. In the Australian guideline, cefazolin is considered as the primary choice of cephalosporins for SAP. However, in our study, none of the patients were treated with respective drug. ceftriaxone and cefoperazone were the most often drugs prescribed. About $55.1 \%$ of cefoperazone and $28 \%$ of ceftriaxone included in both pre- and post-operative prescription. Sixty participants were given ceftriaxone only as surgical prophylaxis, despite the fact that Australian guideline does not recommended it for any procedures. Ceftriaxone is a broadspectrum antibiotic; therefore, its use as SAP would rise to the emergence of resistance and would either lead to a lack of response for any infections.

A study conducted in Ayder Referral hospital reported that the total compliance to SAP guidelines was $25 \%$. Majority of the non-compliance was inappropriate SAP selection and extending the duration of antibiotic prophylaxis to more than $24 \mathrm{~h}$. About $19.4 \%$ of procedures were non-compliant to SAP guideline in terms of indications.

Mousavi et al. [8] conducted an "audit of perioperative antimicrobial prophylaxis: compliance with international guideline" reported that in her study, only $22 \%$ of cases are under compliance with all recommendations.

Table 5: Risk factor associated with SSI

\begin{tabular}{|c|c|c|c|c|c|}
\hline S. No. & Risk factors & Category & $\begin{array}{l}\text { Number of } \\
\text { patients (178) }\end{array}$ & $\begin{array}{l}\text { Frequency } \\
\text { of SSI (11) }\end{array}$ & Percentage \\
\hline \multirow[t]{7}{*}{1.} & \multirow[t]{7}{*}{ Age } & $0-12$ & 7 & 0 & 0 \\
\hline & & $13-18$ & 12 & 1 & 8.33 \\
\hline & & $19-35$ & 47 & 2 & 4.2 \\
\hline & & $36-50$ & 40 & 2 & 5 \\
\hline & & $51-65$ & 49 & 4 & 8.1 \\
\hline & & $66-84$ & 21 & 2 & 9.52 \\
\hline & & $\geq 85$ & 2 & 0 & 0 \\
\hline \multirow[t]{2}{*}{2.} & \multirow[t]{2}{*}{ Gender } & Male & 100 & 7 & 7 \\
\hline & & Female & 78 & 4 & 5.12 \\
\hline \multirow[t]{6}{*}{3.} & \multirow[t]{6}{*}{ Comorbidity } & DM only & 19 & 4 & 21.05 \\
\hline & & HTN only & 8 & 2 & 25 \\
\hline & & $\mathrm{DM}+\mathrm{HTN}$ & 24 & 0 & 0 \\
\hline & & $\mathrm{DM}+\mathrm{HTN}+$ Necrotising fasciculitis, anemia & 3 & 0 & 0 \\
\hline & & Miscellaneous & 11 & 0 & 0 \\
\hline & & No-comorbidity & 113 & 5 & 4.42 \\
\hline \multirow[t]{6}{*}{4.} & \multirow{6}{*}{ Procedure } & Wound debridement and secondary suturing & 10 & 4 & 40 \\
\hline & & CABG and post-CABG infection & 5 & 2 & 40 \\
\hline & & TKR & 3 & 2 & 66.66 \\
\hline & & Emergency LSCS & 18 & 1 & 5.55 \\
\hline & & TAH & 15 & 1 & 6.66 \\
\hline & & I and D & 4 & 1 & 25 \\
\hline \multirow[t]{4}{*}{5.} & \multirow[t]{2}{*}{ Pre-operative } & Antibiotics received & 162 & 9 & 5.55 \\
\hline & & Not received & 16 & 2 & 12.5 \\
\hline & \multirow[t]{2}{*}{ Post-operative } & Antibiotics received & 168 & 11 & 6.54 \\
\hline & & Not received & 10 & 0 & 0 \\
\hline \multirow[t]{3}{*}{6.} & \multirow[t]{3}{*}{ Antiseptic wash } & Povidone-iodine only & 154 & 9 & 5.84 \\
\hline & & Povidone-iodine+NS & 13 & 1 & 7.69 \\
\hline & & NS only & 7 & 1 & 14.28 \\
\hline \multirow[t]{6}{*}{7.} & Antibiotic & Inappropriate drug & 151 & 11 & 7.28 \\
\hline & \multirow{5}{*}{ administration } & Appropriate drug & 21 & 0 & 0 \\
\hline & & Inappropriate dose & 57 & 6 & 10.52 \\
\hline & & Appropriate dose & 115 & 5 & 4.34 \\
\hline & & Inappropriate duration & 126 & 8 & 6.34 \\
\hline & & Appropriate duration & 46 & 3 & 6.52 \\
\hline
\end{tabular}

SSI: Surgical site infection, DM: Diabetes mellitus, HTN: Hypertension, CABG: Coronary artery bypass graft, TKR: Total knee replacement, LSCS: Lower segment cesarean section, NS: Normal saline, TAH: Total abdominal hysterectomy 
Among the total population, around 11 patients had SSI due to inappropriate antibiotic selection, administration, and non-compliance to SAP guidelines. The risk factors associated with SSI are mentioned in Table 5 .

The presence of comorbidity also had influence in SSI. About 25\% of patients with SSI had HTN as comorbid condition. The highest rate of SSI was in the patients admitted for total knee replacement (66.66\%). This was mainly due to inappropriate care and omission of pre-operative antibiotic drug. While considering drug administration, 11 cases with SSI were treated with inappropriate drug and majority with inappropriate duration and inappropriate dose. Hence, surgical antibiotic prophylaxis plays a major role in preventing the occurrence of SSI

The study conducted by Ahmed et al. [9] mentioned that the rate of SSI is more in patients receiving post-operative antibiotics.

\section{Limitations of the study}

The key limitation of our study was difficult to explain about SAP importance and requirement because hospital authority was not ready to accept their fault. Post-discharge monitoring after the first review was not conducted by outpatient clinics, so the determination of SSI occurred after the first review was not done because SSI may emerge after 10 days. In some cases, patients were referred to other hospitals, so in that cases, completion of data collection was not possible.

\section{CONCLUSION}

The present study revealed that there is a poor compliance to the SAP guidelines followed in the respective hospital in terms of inappropriate antibiotic selection, inappropriate duration of antibiotic both pre-and postoperative, and prescribing of resistant drug. A total of 178 patients were observed in this study, of which 11 affected with SSI due to inappropriate antibiotic selection and in appropriate timing of drug administration and omission of pre-operative drug. This is mainly due to the unavailability of clinical pharmacist to assist physician in the selection and administration of the correct choice of prophylactic antibiotic according to the guideline. Another reason was that medical residents were not as fully trained on medication choices. Clinical pharmacist can solve these by providing proper counseling to the staff about SAP importance and its requirement in preventing SSI. In this study, we followed the Australian guideline for surgical antibiotic prophylaxis because of unavailability of proper national or local SAP guideline. This study emphasizes that there is dire need to make local SAP guidelines and dissemination of that among all health-care workers to improve the antibiotic-prescribing pattern and patient safety by reducing the occurrence of SSI

\section{ACKNOWLEDGMENTS}

We express our heartfelt thanks to Dr. D. Krishnarajan, M. Pharm., Ph.D (Professor and HOD) and Mr. K. C. Arul Prakasam, M. Pharm., Mr. A. Srinivasan, M. Pharm., Associate professors for their great support and kindly granting the permission to forward our study.

\section{AUTHORS' CONTRIBUTION}

Dr. N. Senthil Kumar: Manuscript review,and statistical analysis. Dr. K. Sivasakthi: Manuscript review, statistical analysis, and final review. Ms. Jisa Elizabeth Joseph: Manuscript preparation, protocol designing, data collection, statistical analysis, and report preparation. Mr. V. A. Vishnukumar: Concept, literature search, data collection, and manuscript preparation. Mr. Geolin. R. P: Data collection, concept, and literature search.

\section{CONFLICTS OF INTERESTS}

Nothing to disclose.

\section{REFERENCES}

1. Rehan HS, Kakkar AK, Goel S. Surgical antibiotic prophylaxis in a tertiary care teaching hospital in India. Int J Infect Control 2010;6:1-6.

2. Mohamoud SA, Yesuf TA, Sisay EA. Utilization assessment of surgical antibiotic prophylaxis at ayder referral hospital. J Appl Pharm 2016; 8:1-5.

3. Vessal G, Namazi S, Davarpanah MA, Foroughinia F. Evaluation of prophylactic antibiotic administration at the surgical ward of a major referral hospital, islamic republic of Iran. East Mediterr Health J 2011;17:663-7.

4. Government of South Australia, Department of Health. Surgical Antibiotic Prophylaxis clinical Guidelines. Australia: Government of South Australia, Department of Health; 2017. p. 1-44.

5. Tripathi KD. Essentials of Medical Pharmacology. $8^{\text {th }}$ ed. New Delhi: Jaypee Brothers Medical Publishers (P) Ltd.

6. Sharma P, Goel D. Utilization assessment of antimicrobial prophylaxis in surgical patients at tertiary care teaching hospital. Saudi J Health Sci 2018;7:23-6

7. Vessal G, Namazi S, Davarpanah MA, Foroughinia F. Evaluation of prophylactic antibiotic administration at the surgical ward of a major referral hospital, islamic republic of Iran. East Mediterr Health J 2011;17:663-7.

8. Mousavi S, Zamani E, Bahrami F. An audit of perioperative antimicrobial prophylaxis: Compliance with the international guidelines. J Res Pharm Pract 2017;6:1-4.

9. Ahmed SO, Mahadi SI, Ahmed M. Antibiotic prophylaxis in clean and clean-contaminated surgery and surgical site infection in Khartoum teaching hospital. Sudan Med J 2015;50:24-32. 